\title{
REAL HYPERSURFACES IN A COMPLEX SPACE FORM WITH A CONDITION ON THE STRUCTURE JACOBI OPERATOR
}

\author{
S. H. Kon - TeE-How Loo - ShIQUAN ReN \\ (Communicated by Július Korbaš)
}

\begin{abstract}
In this paper we classify the real hypersurfaces in a non-flat complex space form with its structure Jacobi operator $R_{\xi}$ satisfying $\left(\nabla_{X} R_{\xi}\right) \xi=0$, for all vector fields $X$ in the maximal holomorphic distribution $D$. With this result, we prove the non-existence of real hypersurfaces with $D$-parallel as well as $D$-recurrent structure Jacobi operator in complex projective and hyperbolic spaces. We can also prove the non-existence of real hypersurfaces with recurrent structure Jacobi operator in a non-flat complex space form as a corollary.
\end{abstract}

(C) 2014

Mathematical Institute

Slovak Academy of Sciences

\section{Introduction}

Let $M_{n}(c)$ be an $n$-dimensional non-flat complex space form with constant holomorphic sectional curvature $4 c$. It is known that a complete and simply connected non-flat complex space form is either a complex projective space $(c>0)$, denoted by $\mathbb{C} P^{n}$, or a complex hyperbolic space $(c<0)$, denoted by $\mathbb{C} H^{n}$. Without loss of generality, we always assume $c=1$ for $\mathbb{C} P^{n}$ and $c=-1$ for $\mathbb{C} H^{n}$.

Let $M$ be a real hypersurface in $M_{n}(c)$. The Jacobi operator $R_{X}$, with respect to a tangent vector field $X$ on an open subset of $M$, is defined by $R_{X}(Y)=$ $R(Y, X) X$, for vector fields $Y$ tangent to $M$, where $R$ is the curvature tensor

2010 Mathematics Subject Classification: Primary 53C15; Secondary 53B25.

Keywords: complex space forms, structure Jacobi operator, real hypersurfaces, ruled real hypersurfaces.

The first and second authors were partially supported by Grant UMRG (No. RG163/11AFR).

The third author was partially supported by Grant UM.TNC2/IPPP (No. PS030/2011A). 


\section{S. H. KON - TEE-HOW LOO - SHIQUAN REN}

on $M$. In particular, for the structure vector field $\xi=-J N$, where $N$ is locally a unit normal vector field of $M, R_{\xi}$ is called the structure Jacobi operator of $M$. Let $D$ denote the distribution determined by tangent vectors perpendicular to $\xi$ at each point of $M . D$ is called the maximal holomorphic distribution on $M$. If the structure Jacobi operator satisfies $\left(\nabla_{X} R_{\xi}\right) Y=0$, for any vector field $X$ in $D$ and $Y$ tangent to $M$, then it is said to be $D$-parallel; and if it satisfies $\left(\nabla_{X} R_{\xi}\right) Y=\omega(X) R_{\xi}(Y)$ for any vector field $X$ in $D$ and $Y$ tangent to $M$, where $\omega$ is a 1 -form on $M$, then the structure Jacobi operator is said to be $D$-recurrent.

In [5], J. D. Pérez, F. G. Santos and Y. J. Suh studied the non-existence of real hypersurfaces with $D$-parallel structure Jacobi operator in a complex projective space, leaving the complex hyperbolic case open. The concept of $D$-recurrent structure Jacobi operator was first considered in [6], which gives the original idea to our paper. However, a part of the proof given for the classification of real hypersurfaces in $\mathbb{C} P^{n}$ with its structure Jacobi operator $D$-recurrent $([6$; $§ 5$, p. 221]) cannot be justified. Actually, there does not exist any real hypersurface in $M_{n}(c)$, for $n>2$, with $D$-recurrent structure Jacobi operator as will be shown.

Recently T. Theofanidis and P. J. Xenos proved in [8] that there does not exist any real hypersurface in $M_{n}(c), n>2$, with recurrent structure Jacobi operator. They also studied real hypersurfaces in $M_{2}(c)$ with $D$-recurrent structure Jacobi operator in [9].

In this article, we consider a condition weaker than $D$-parallelism and $D$-recurrence on the structure Jacobi operator and prove the following theorem.

TheOREM 1.1. Let $M$ be a real hypersurface in $M_{n}(c), n>2$, satisfying $\left(\nabla_{X} R_{\xi}\right) \xi=0$, for all vector fields $X$ in $D$. Then $M$ is locally congruent to a ruled real hypersurface.

The above theorem will lead to the proof of non-existence of real hypersurfaces with $D$-parallel or $D$-recurrent structure Jacobi operator in $M_{n}(c)$.

THEOREM 1.2. There does not exist any real hypersurface $M$ in $M_{n}(c), n>2$, with its structure Jacobi operator D-recurrent: $\left(\nabla_{X} R_{\xi}\right) Y=\omega(X) R_{\xi} Y$, for all vector fields $X$ in $D$ and $Y$ tangent to $M$. Here $\omega$ denotes a 1-form on $M$.

From Theorem 1.2, we have the following results.

COROLlARY 1.3. There does not exist any real hypersurface $M$ in $M_{n}(c), n>2$, with its structure Jacobi operator D-parallel: $\left(\nabla_{X} R_{\xi}\right) Y=0$, for all vector fields $X$ in $D$ and $Y$ tangent to $M$.

COROLlARY 1.4. There does not exist any real hypersurface $M$ in $M_{n}(c), n>2$, with recurrent structure Jacobi operator, i.e., $\nabla R_{\xi}=\omega \otimes R_{\xi}$. 


\section{REAL HYPERSURFACES IN A COMPLEX SPACE FORM}

\section{Preliminaries}

Throughout this paper, all manifolds, vector fields, etc., will be considered of class $C^{\infty}$ unless otherwise stated. Let $M$ be a connected real hypersurface in $M_{n}(c), n>2$, without boundary. Let $N$ be a locally defined unit normal vector field on $M$. Denote by $\nabla$ the Levi-Civita connection on $M$ induced from $M_{n}(c)$. Let $\langle\cdot, \cdot\rangle$ denote the Riemannian metric of $M$ induced from the Riemannian metric of $M_{n}(c)$ and $A$ be the shape operator of $M$ in $M_{n}(c)$. Now, we define a tensor field $\phi$ of type $(1,1)$, a vector field $\xi$ and a 1-form $\eta$ by

$$
J X=\phi X+\eta(X) N, \quad J N=-\xi .
$$

Then it is seen that $\langle\xi, X\rangle=\eta(X)$. Furthermore, the set of tensors $(\phi, \xi, \eta,\langle\cdot, \cdot\rangle)$ is an almost contact metric structure on $M$, i.e., they satisfy the following

$$
\phi^{2} X=-X+\eta(X) \xi, \quad \phi \xi=0, \quad \eta(\phi X)=0, \quad \eta(\xi)=1 .
$$

From the parallelism of $J$ we get

$$
\left(\nabla_{X} \phi\right) Y=\eta(Y) A X-\langle A X, Y\rangle \xi
$$

and

$$
\nabla_{X} \xi=\phi A X .
$$

Let $R$ be the curvature tensor of $M$. Then the Gauss and Codazzi equations are respectively given by

$$
\begin{array}{r}
R(X, Y) Z=c\{\langle Y, Z\rangle X-\langle X, Z\rangle Y+\langle\phi Y, Z\rangle \phi X-\langle\phi X, Z\rangle \phi Y \\
-2\langle\phi X, Y\rangle \phi Z\}+\langle A Y, Z\rangle A X-\langle A X, Z\rangle A Y, \\
\left(\nabla_{X} A\right) Y-\left(\nabla_{Y} A\right) X=c\{\eta(X) \phi Y-\eta(Y) \phi X-2\langle\phi X, Y\rangle \xi\} .
\end{array}
$$

From the Gauss equation, we have

$$
\begin{gathered}
R_{\xi} Y=c\{Y-\eta(Y) \xi\}+\alpha A Y-\eta(A Y) A \xi \\
\left(\nabla_{X} R_{\xi}\right) Y=-c\langle Y, \phi A X\rangle \xi-c \eta(Y) \phi A X+(X \alpha) A Y+\alpha\left(\nabla_{X} A\right) Y \\
-\left\langle\left(\nabla_{X} A\right) Y, \xi\right\rangle A \xi-\langle Y, A \phi A X\rangle A \xi \\
-\eta(A Y)\left(\nabla_{X} A\right) \xi-\eta(A Y) A \phi A X
\end{gathered}
$$

for all vector fields $X, Y$ tangent to $M$.

For a unit vector field $U$ in $D$, where $D$ is the maximal holomorphic distribution, let $D_{U}$ denote the collection of tangent vectors orthogonal to $\xi, U$ and $\phi U$ at each point of $M$. Then according to our assumption $n>2, D_{U}$ is a subdistribution of $D$ with a positive dimension. Denote $\alpha=\langle A \xi, \xi\rangle$ and $\beta=\|\phi A \xi\|$. 


\section{S. H. KON - TEE-HOW LOO - SHIQUAN REN}

A real hypersurface is said to be Hopf if $A \xi=\alpha \xi$. A ruled real hypersurface is a non-Hopf real hypersurface satisfying the condition $\langle A X, Y\rangle=0$, for all vector fields $X, Y$ in $D$. A real hypersurface is ruled if and only if its shape operator could be expressed as $A \xi=\alpha \xi+\beta U, A U=\beta \xi, A X=0$ for $X \perp \operatorname{span}\{\xi, U\}$, $\langle U, U\rangle=1$ and $\beta \neq 0$ on an open dense subset.

We recall the following results for later use.

TheOREM 2.1. ([3]) Let $M$ be a Hopf hypersurface in $M_{n}(c), n>1$, and let $X$ be in D. If $A X=\lambda X$, and $A \phi X=\lambda \phi X$, then $\lambda^{2}=\alpha \lambda+c$.

LEMма 2.2. ([1]) Let $M$ be a real hypersurface in $M_{n}(c), n>2$. Suppose $\langle(\phi A-A \phi) X, Y\rangle=0$ for all vector fields $X, Y$ in $D$. Let $G_{1}=\{x \in M$ : $\left.\|\phi A \phi\|_{x} \neq 0\right\}$. Then on $G_{1}$, we have grad $\alpha=\alpha V-2 A V$, where $V=\phi A \xi$. Furthermore, if we suppose $A \xi=\alpha \xi+\beta U$, where $U$ is a unit vector field in $D$, and $\beta$ is a nonvanishing function on $G_{1}$, and $A V=0$, then we have $\operatorname{grad} \beta=$ $\left(c+\beta^{2}\right) \phi U$.

Lemмa 2.3. ([2], 7]) If $M$ is a ruled real hypersurface in $M_{n}(c), n>2$, then we have

$$
\left(\nabla_{X} A\right) Y=\{-c\langle\phi X, Y\rangle+\eta(A Y)\langle X, V\rangle+\eta(A X)\langle Y, V\rangle\} \xi
$$

for all tangent vectors $X, Y$ in $D$, where $V=\phi A \xi$.

\section{Some lemmas}

We begin with some lemmas in preparation for the proof of Theorem 1.1 ,

Lemмa 3.1. Let $M$ be a non-Hopf real hypersurface in $M_{n}(c), n>2$. Suppose $M$ satisfies $A \xi=\alpha \xi+\beta U$, where $\beta$ is non-vanishing and $U$ is a unit vector field in $D$, and there exists a unit vector field $Z$ in $D_{U}=\{X \in T M: X \perp \xi, U, \phi U\}$ such that $A Z=\lambda Z$ and $A \phi Z=\lambda \phi Z$.

(a) If $M$ satisfies

$$
A \phi U=\delta \phi U
$$

then

$$
(\lambda-\delta)\left(\lambda^{2}-\alpha \lambda-c\right)=\beta \phi U \lambda .
$$

(b) If $M$ satisfies

$$
A U=\beta \xi+\gamma U
$$

then

$$
(\lambda-\gamma)\left(\lambda^{2}-\alpha \lambda-c\right)-\beta^{2} \lambda=0
$$




\section{REAL HYPERSURFACES IN A COMPLEX SPACE FORM}

(c) If $M$ satisfies both (3.1) and (3.3) then

$$
\beta \lambda(\lambda-\delta)-(\lambda-\gamma) \phi U \lambda=0 .
$$

Pr o of. Suppose $M$ satisfies (3.1). Taking inner product in the Codazzi equation

$$
\left(\nabla_{Z} A\right) \xi-\left(\nabla_{\xi} A\right) Z=-c \phi Z
$$

with $\phi Z$, we obtain

$$
\beta\left\langle\nabla_{Z} U, \phi Z\right\rangle=\lambda^{2}-\alpha \lambda-c .
$$

Taking inner product in the Codazzi equation

$$
\left(\nabla_{Z} A\right) \phi U-\left(\nabla_{\phi U} A\right) Z=0
$$

with $Z$, we obtain

$$
(\delta-\lambda)\left\langle\nabla_{Z} \phi U, Z\right\rangle=\phi U \lambda
$$

By using

$$
\nabla_{Z} \phi U=\phi \nabla_{Z} U
$$

we have (3.2).

Next, suppose $M$ satisfies (3.3). Taking inner product in the Codazzi equation $\left(\nabla_{Z} A\right) \phi Z-\left(\nabla_{\phi Z} A\right) Z=-2 c \xi$ with $\xi$ and $U$ respectively, we obtain

$$
\begin{gathered}
\left\langle\nabla_{\phi Z} Z-\nabla_{Z} \phi Z, U\right\rangle=\frac{2\left(\lambda^{2}-\alpha \lambda-c\right)}{\beta}, \\
(\lambda-\gamma)\left(\left\langle\nabla_{\phi Z} Z, U\right\rangle-\left\langle\nabla_{Z} \phi Z, U\right\rangle\right)=2 \beta \lambda .
\end{gathered}
$$

Combining these two equations, we obtain (3.4).

Finally, if $M$ satisfies both (3.1) and (3.3) then by using (3.2) and (3.4), we get (3.5).

It is stated in 4] that there exist no real hypersurfaces $M$ in $\mathbb{C} P^{n}, n>2$, with shape operator given by $A \xi=\xi+\beta U, A U=\beta \xi+\left(\beta^{2}-1\right) U, A X=-X$ for all $X \perp \xi, U$, where $U$ is a unit vector field in $D$ and $\beta$ is a nonvanishing function. We shall generalize this statement to $M_{n}(c)$ and give an alternative proof.

LEMMA 3.2. Suppose $M$ is a real hypersurface in $M_{n}(c), n>1$, such that the shape operator satisfies $A \xi=c \xi+\beta U, A U=\beta \xi+\left(\beta^{2}-c\right) U, A \phi U=-c \phi U$, where $U$ is a unit vector field in $D$ and $\beta$ is a nonvanishing function defined on $M$. Then $c>0$. Furthermore, if $n>2$, then there exists a vector field $X$ in $D_{U}$ such that $A X \neq-X$. 


\section{S. H. KON - TEE-HOW LOO - SHIQUAN REN}

P r o of. Taking inner product in the Codazzi equation $\left(\nabla_{U} A\right) \phi U-\left(\nabla_{\phi U} A\right) U=$ $-2 c \xi$ with $U$ and $\xi$ respectively, we obtain

$$
\begin{array}{r}
-\beta\left\langle\nabla_{U} \phi U, U\right\rangle+\beta^{2}-3 c-2 \phi U \beta=0, \\
-\beta\left\langle\nabla_{U} \phi U, U\right\rangle+3 c \beta^{2}-4 c^{2}+2 c-\phi U \beta=0 .
\end{array}
$$

From these two equations we obtain

$$
\beta^{2}-3 c \beta^{2}+4 c^{2}-5 c-\phi U \beta=0 .
$$

Taking inner product in the Codazzi equation $\left(\nabla_{\phi U} A\right) \xi-\left(\nabla_{\xi} A\right) \phi U=c U$ with $U$ and $\xi$ respectively, we obtain

$$
\begin{aligned}
\beta^{2}\left\langle\nabla_{\xi} \phi U, U\right\rangle+2 c^{2}-c \beta^{2}-\beta^{2}-c+\phi U \beta & =0, \\
\left\langle\nabla_{\xi} \phi U, U\right\rangle-4 c & =0 .
\end{aligned}
$$

From these two equations we obtain

$$
3 c \beta^{2}+2 c^{2}-\beta^{2}-c+\phi U \beta=0 .
$$

By summing up (3.8) and (3.10), we obtain

$$
c(c-1)=0,
$$

which cannot happen when $c=-1$. Hence $c=1$ and (3.8) becomes

$$
\phi U \beta=-2 \beta^{2}-1 \text {. }
$$

From (3.7) and (3.11), we have

$$
\beta\left\langle\nabla_{U} \phi U, U\right\rangle=5 \beta^{2}-1
$$

From (3.9) we have

$$
\left\langle\nabla_{\xi} \phi U, U\right\rangle=4
$$

Now suppose to the contrary that $A X=-X$ for any vector field $X$ in $D_{U}$. For any unit vector field $Z$ in $D_{U}$, taking inner product in the Codazzi equation $\left(\nabla_{Z} A\right) \xi-\left(\nabla_{\xi} A\right) Z=-\phi Z$ with $U, \phi U, \xi$ respectively, we have

$$
\begin{aligned}
Z \beta+\beta^{2}\left\langle\nabla_{\xi} Z, U\right\rangle & =0, \\
\left\langle\nabla_{Z} U, \phi U\right\rangle & =0, \\
\left\langle\nabla_{\xi} Z, U\right\rangle & =0 .
\end{aligned}
$$

From (3.14) and (3.16), we obtain

$$
Z \beta=0 .
$$

Take inner product in the Codazzi equation $\left(\nabla_{Z} A\right) \phi U-\left(\nabla_{\phi U} A\right) Z=0$ with $\xi$,

$$
\left\langle\nabla_{Z} \phi U, U\right\rangle=\left\langle\nabla_{\phi U} Z, U\right\rangle ;
$$


then apply (3.15),

$$
\left\langle\nabla_{\phi U} U, Z\right\rangle=0 .
$$

Take inner product in the Codazzi equation $\left(\nabla_{Z} A\right) U-\left(\nabla_{U} A\right) Z=0$ with $U$,

$$
2 Z \beta+\beta\left\langle\nabla_{U} Z, U\right\rangle=0 ;
$$

then apply (3.17),

$$
\left\langle\nabla_{U} U, Z\right\rangle=0 .
$$

Taking inner product in the Codazzi equation $\left(\nabla_{U} A\right) \phi U-\left(\nabla_{\phi U} A\right) U=-2 \xi$ with $\phi U$, we have

$$
\left\langle\nabla_{\phi U} U, \phi U\right\rangle=0
$$

From (3.18), (3.20) and $\left\langle\nabla_{\phi U} U, \xi\right\rangle=-1$, we obtain

$$
\nabla_{\phi U} U=-\xi
$$

hence

$$
\nabla_{\phi U} \phi U=0 .
$$

From (3.12), (3.19) and $\left\langle\nabla_{U} U, \xi\right\rangle=0$, we obtain

$$
\nabla_{U} U=\frac{1-5 \beta^{2}}{\beta} \phi U
$$

hence

$$
\nabla_{U} \phi U=\left(1-\beta^{2}\right) \xi+\frac{5 \beta^{2}-1}{\beta} U .
$$

From (3.13), (3.16) and $\left\langle\nabla_{\xi} U, \xi\right\rangle=0$, we obtain

$$
\nabla_{\xi} U=-4 \phi U .
$$

We also have

$$
\nabla_{\phi U} \xi=U .
$$

Let $X=U, Y=\phi U, Z=U$ in the Gauss equation, we have

$$
R(U, \phi U) U=\left(\beta^{2}-5\right) \phi U .
$$

On the other hand, applying (3.11), (3.21), (3.22), (3.23), (3.24), (3.25), (3.26) to

we have

$$
R(U, \phi U) U=\nabla_{U} \nabla_{\phi U} U-\nabla_{\phi U} \nabla_{U} U-\nabla_{[U, \phi U]} U,
$$

$$
R(U, \phi U) U=\left(10 \beta^{2}-8\right) \phi U .
$$

From (3.27) and (3.28), we see that $\beta$ is constant. This contradicts (3.11). Hence the proof is completed. 
S. H. KON - TEE-HOW LOO - SHIQUAN REN

\section{Proof of Theorem 1.1}

In this section we give a classification of real hypersurfaces in $M_{n}(c)$ satisfying the condition

$$
\left(\nabla_{X} R_{\xi}\right) \xi=0
$$

for all vector fields $X$ in $D$. Note that the condition (4.1) is equivalent to

$$
c \phi A X+\alpha A \phi A X-\langle\phi A X, A \xi\rangle A \xi=0,
$$

for any tangent vector field $X$ in $D$. Hence by (4.2), for any vector fields $X, Y$ in $D$,

$$
c\langle(\phi A-A \phi) X, Y\rangle=\langle\phi A X, A \xi\rangle\langle A \xi, Y\rangle+\langle X, A \xi\rangle\langle\phi A Y, A \xi\rangle .
$$

Proposition 4.1. There does not exist any Hopf hypersurface in $M_{n}(c), n>2$, satisfying the condition $\left(\nabla_{X} R_{\xi}\right) \xi=0$ for any vector field $X$ in $D$.

P r o of. Suppose $M$ is such a Hopf hypersurface. Equation (4.3) becomes

$$
\langle(\phi A-A \phi) X, Y\rangle=0
$$

for all vector fields $X, Y$ tangent to $M$. Hence $A \phi=\phi A$. Pointwise, we get that $D_{\lambda}=\{X \in D: A X=\lambda X\}$ is $\phi$-invariant. Hence by Theorem 2.1, we obtain

$$
\lambda^{2}=\alpha \lambda+c .
$$

Let $X$ be a unit principal vector field in $D$ such that $A X=\lambda X$. Then by (4.2), we obtain

$$
\lambda(c+\alpha \lambda)=0 .
$$

From the above two equations, we get $\lambda^{3}=0$. Hence $\lambda=0$ and this contradicts (4.5).

In the rest of this section, let $M$ be a real hypersurface in $M_{n}(c)$ that satisfies the condition (4.1). We also suppose that $A \xi=\alpha \xi+\beta U$ with $\beta \neq 0$ everywhere on $M$ and $U$ a unit vector field in $D$.

From (4.3) we obtain that for any vector fields $X, Y$ in $D$,

$$
c\langle(\phi A-A \phi) X, Y\rangle=-\beta^{2}\{\langle Y, U\rangle\langle\phi U, A X\rangle+\langle X, U\rangle\langle\phi U, A Y\rangle\} .
$$

Proposition 4.2. For a real hypersurface $M$ in $M_{n}(c), n>2$, satisfying the condition (4.1), we have

(a) $A \phi U=\delta \phi U$, where $\delta$ is a function on $M$,

(b) $A U=\beta \xi+\left(1-\frac{\beta^{2}}{c}\right) \delta U$,

(c) $\left(\beta^{2}-c\right)(c+\alpha \delta) \delta=0$. 


\section{REAL HYPERSURFACES IN A COMPLEX SPACE FORM}

Pr o o f. Let $X=Y=\phi U$ in (4.6), then we have

$$
\langle A U, \phi U\rangle=0 .
$$

If we let $X=U$ and $Y$ an arbitrary vector field in $D$ in (4.6), then

$$
c\langle\phi A U-A \phi U, Y\rangle=-\beta^{2}\langle A \phi U, Y\rangle .
$$

Since (4.8) also holds for $Y=\xi$, we obtain

$$
c A \phi U-c \phi A U=\beta^{2} A \phi U .
$$

By putting $X=\phi U$ and replacing $Y$ with $\phi Y$ in (4.6), we have

$$
c\langle A \phi U-\phi A U, Y\rangle=\beta^{2}\langle A \phi U, \phi U\rangle\langle\phi U, Y\rangle,
$$

for any vector field $Y$ in $D$. Since (4.10) also holds for $Y=\xi$, we obtain

$$
c A \phi U-c \phi A U=\beta^{2}\langle A \phi U, \phi U\rangle \phi U .
$$

Putting $X=\phi U$ in (4.2) and taking inner product with $U$, we get

$$
\left(c-\beta^{2}\right)\langle\phi A \phi U, U\rangle+\alpha\langle A \phi A \phi U, U\rangle=0 .
$$

From (4.9) and (4.11), we get (a) and (b). From (a), (b) and (4.12), we get (c).

From this proposition we know that $D_{U}$ is invariant under $A$. In particular, for any vector fields $X, Y$ in $D_{U}$, (4.6) becomes

$$
\langle(\phi A-A \phi) X, Y\rangle=0 .
$$

Therefore, let $D_{\lambda}=\left\{X \in D_{U}: A X=\lambda X\right\}$ denote a pointwise subspace of $D_{U}$; then $D_{\lambda}$ is $\phi$-invariant.

Let $Y$ be a unit vector field in $D_{U}$ satisfying $A Y=\lambda Y$. From (4.2) we have

$$
\lambda(c+\alpha \lambda)=0 .
$$

From (4.14), we consider the following two cases when $M$ is non-Hopf.

CASE 1. $A=0$ on $D_{U}$.

Hence $D_{U}=D_{0}$ at each point of $M$, i.e., $A Y=0$ for any vector field $Y$ in $D_{U}$. From (3.2), we have $\delta=0$. Therefore, by Proposition 4.2. $M$ satisfies $A \xi=\alpha \xi+\beta U, A U=\beta \xi, A X=0$, for all vector fields $X$ perpendicular to $\xi$ and $U$. Hence $M$ is a ruled real hypersurface.

CASE 2. $A \neq 0$ on $D_{U}$.

In this case, there exists a unit vector field $Y$ in $D_{U}$, such that $A Y=\lambda_{1} Y$, where $\lambda_{1} \neq 0$ on an open subset of $M$. We identify this subset with $M$. By (4.14), $\alpha \neq 0$ and $\lambda_{1}=-c / \alpha$. From (3.4), we get

$$
\alpha^{2} \beta^{2}=\alpha\left(c-\beta^{2}\right) \delta+c^{2} .
$$


By applying (4.15) to Proposition 4.2 (c), we get

$$
\delta\left(\alpha^{2}-c\right)=0
$$

We shall consider the following two subcases.

SubCASE 2-A. $\delta \neq 0$ at some point of $M$.

By continuity, there exists an open subset of $M$, such that on this open subset, $\delta \neq 0$ at each point. From (4.16), $\alpha^{2}=c$ on this open subset. If necessary, we replace the normal vector field $N$ by $-N$, so that $\alpha=c=1$. Then $\lambda_{1}=-1$ on this open subset. Putting $\lambda=-1$ in (3.2), we obtain $\delta=-1$. From (4.14), for any principal unit vector field $Y$ in $D_{U}$ such that $A Y=\lambda Y, \lambda(\lambda+1)=0$. Hence by continuity, $\lambda$ is constantly 0 or -1 . By using (3.2), we see that $\lambda=-1$. This subcase cannot happen according to Lemma 3.2 and Proposition 4.2.

SubCASE 2-B. $\delta=0$ at every point of $M$. By using $\delta=0$ and (4.6), we obtain $\langle(\phi A-A \phi) X, Y\rangle=0$ for all $X, Y$ in $D$. We use the same notation $G_{1}$ as in Lemma 2.2. By continuity of the norm, $G_{1}$ is an open subset of $M$. On $G_{1}$, by using Lemma 2.2, we have

$$
\phi U \alpha=\alpha \beta
$$

and

$$
\phi U \beta=\beta^{2}+c .
$$

From (4.15), we have $\alpha^{2} \beta^{2}=c^{2}$; then take the covariant derivative in the direction of $\phi U$,

$$
\beta(\phi U \alpha)+\alpha(\phi U \beta)=0 .
$$

Putting (4.17), (4.18) into (4.19), with the help of $\alpha \neq 0$, we get

$$
2 \beta^{2}+c=0
$$

Hence $\beta$ is constant and by (4.18), we have $\beta^{2}+c=0$. This is a contradiction if $G_{1}$ is non-empty.

From the above argument we have $G_{1}$ must be empty and $\phi A \phi=0$ must hold everywhere on $M$, hence $M$ is a ruled real hypersurface. But this contradicts $D_{-c / \alpha} \neq 0$, which holds in the whole Case 2. So Subcase 2-b is impossible.

Now we have proved that if $M$ is a real hypersurface in $M_{n}(c)$ satisfying $\left(\nabla_{X} R_{\xi}\right) \xi=0$, for all vector fields $X$ in $D$, then the only possibility for $M$ is that it is a ruled real hypersurface. Conversely, it is easy to check that ruled real hypersurfaces satisfy (4.2). So we have completed the proof of Theorem 1.1. 


\section{REAL HYPERSURFACES IN A COMPLEX SPACE FORM}

\section{Proof of Theorem 1.2}

We only need to verify that the structure Jacobi operator $R_{\xi}$ of ruled real hypersurfaces cannot be $D$-recurrent. Suppose there exists a ruled real hypersurface with its structure Jacobi operator $D$-recurrent. Then its shape operator satisfies $\langle A X, Y\rangle=0$, for vector fields $X, Y$ in $D$. From Lemma 2.3, it also satisfies $\left\langle\left(\nabla_{X} A\right) Y, Z\right\rangle=0$ for all vector fields $X, Y$ and $Z$ in $D$.

We consider $X, Y$ in $D$ for (2.2). Taking inner product on both sides of (2.2) with a unit tangent vector $Z$ in $D_{U}$, and applying (2.1), we obtain

$$
-\eta(A Y)\left\langle\left(\nabla_{X} A\right) Z, \xi\right\rangle=c \omega(X)\langle Y, Z\rangle .
$$

It follows from Lemma 2.3 that this equation becomes

$$
c \eta(A Y)\langle\phi X, Z\rangle=c \omega(X)\langle Y, Z\rangle .
$$

By putting $Y=U$ and $X=\phi Z$ in the above equation, we obtain $\beta=0$, which is a contradiction. Hence such a ruled real hypersurface cannot exist.

Remark 5.1. From the proof of Theorem 1.2 we get the following result: In $M_{n}(c), n>2$, there does not exist a ruled real hypersurface with its structure Jacobi operator $\eta$-recurrent, i.e., $\left\langle\left(\nabla_{X} R_{\xi}\right) Y, Z\right\rangle=$ $\omega(X)\left\langle R_{\xi} Y, Z\right\rangle$ for all vector fields $X, Y, Z$ in $D$.

Acknowledgement. The present authors would like to express their deep gratitude to the referee for the careful reading of our manuscript and valuable comments to improve the paper.

\section{REFERENCES}

[1] KON, S. H.-TEE-HOW LOO: On a class of real hypersurfaces in a complex space form. Preprint.

[2] KON, S. H.-TEE-HOW LOO: On characterizations of real hypersurfaces in a complex space form with $\eta$-parallel shape operator, Canad. Math. Bull. 55 (2012), 114-126.

[3] NIEBERGALL, R.--RYAN, P. J.: Real hypersurfaces in complex space forms. Tight and taut submanifolds. In: Math. Sci. Res. Inst. Publ. 32, Cambridge Univ. Press, Cambridge, 1997, pp. 233-304.

[4] ORTEGA, M.-PÉREZ, J. D.-SANTOS, F. G.: Non-existence of real hypersurfaces with parallel structure Jacobi operator in non-flat complex space forms, Rocky Mountain J. Math. 36 (2006), 1603-1613.

[5] PÉREZ, J. D.-SANTOS, F. G.-SUH, Y. J.: Real hypersurfaces in complex projective space whose structure Jacobi operator is D-parallel, Bull. Belg. Math. Soc. Simon Stevin 13 (2006), 459-469. 


\section{S. H. KON - TEE-HOW LOO - SHIQUAN REN}

[6] PÉREZ, J. D.-SANTOS, F. G.: Real hypersurfaces in complex projective space with recurrent structure Jacobi operator, Differential Geom. Appl. 26 (2008), 218-223.

[7] SUH, Y. J.: Characterizations of real hypersurfaces in complex space forms in terms of Weingarten map, Tsukuba J. Math. 14 (1990), 27-37.

[8] THEOFANIDIS, T.-XENOS, P. J.: Non-existence of real hypersurfaces equipped with recurrent structure Jacobi operator in nonflat complex space forms, Results Math. 61 (2012), 43-55.

[9] THEOFANIDIS, T.-XENOS, P. J.: Non-existence of real hypersurfaces equipped with recurrent structure Jacobi operator in nonflat complex space planes, Beitr. Algebra Geom. 53 (2012), 235-246.

Received 10. 2. 2012

Institute of Mathematical Sciences

Accepted 19. 5. 2012

University of Malaya

50603 Kuala Lumpur

MALAYSIA

E-mail: shkon@um.edu.my looth@um.edu.my renshiquan@gmail.com 